\title{
12. Collision between the Two Columnar Objects. II
}

\section{Kinematics after the Collision}

\author{
By Genrokuro NishimURA \\ Tokai University \\ (Communicated by Koji HidAKA, M. J. A., Jan. 12, 1980)
}

Using the same notation and the same analytical method as in paper (I), the velocities of $x$-direction of both objects $\left(M_{1}, M_{2}\right)$ subsequent to the collision are obtained as follows:

For the case when $\left(c_{1} / c_{2} \cdot l_{2} / l_{1}\right) \geqq 1$ and $0 \leqq M_{1} / M_{2} \leqq 1$,

$$
\frac{d U_{1}}{d t}=V_{0}\left(1-2 \alpha_{1}\right), \quad \frac{d U_{2}}{d t}=V_{0} \cdot 2 \alpha_{1} \frac{M_{1}}{M_{2}} .
$$

And further, when $\left(c_{2} / c_{1} \cdot l_{1} / l_{2}\right) \geqq 1$ and $0 \leqq M_{2} / M_{1} \leqq 1$,

$$
\frac{d U_{1}}{d t}=V_{0}\left(1-2 \frac{M_{2}}{M_{1}} \alpha_{3}\right), \quad \frac{d U_{2}}{d t}=V_{0} \cdot 2 \alpha_{3} .
$$

In the equations (1), (2), $\alpha_{1}$ and $\alpha_{3}$ are respectively shown by the following expressions:

$$
\begin{aligned}
\alpha_{1}=\left[\frac{7}{6}+\right. & \frac{M_{1}}{M_{2}}\left\{1+\frac{1}{6}\left(\frac{c_{1}}{c_{2}} \frac{l_{2}}{l_{1}}\right)^{2}\right. \\
& \left.\left.-\frac{1}{2 \pi^{3}}\left(\frac{c_{1}}{c_{2}} \frac{l_{2}}{l_{1}}\right)^{3} \sum_{\nu=1}^{\infty} \frac{1}{\nu^{3}} \sin \left(2 \nu \pi \frac{c_{2}}{c_{1}} \frac{l_{1}}{l_{2}}\right)\right\}\right]^{-1}, \\
\alpha_{3}=\left[\frac{7}{6}+\right. & \frac{M_{2}}{M_{1}}\left\{1+\frac{1}{6}\left(\frac{c_{2}}{c_{1}} \frac{l_{1}}{l_{2}}\right)^{2}\right. \\
& \left.\left.-\frac{1}{2 \pi^{3}}\left(\frac{c_{2}}{c_{1}} \frac{l_{1}}{l_{2}}\right)^{3} \sum_{\nu=1}^{\infty} \frac{1}{\nu^{3}} \sin \left(2 \nu \pi \frac{c_{1}}{c_{2}} \frac{l_{2}}{l_{1}}\right)\right\}\right]^{-1} .
\end{aligned}
$$

For obtaining a concrete understanding of the characteristics of these velocities, the two graphs in Figs. 1 and 2 are obtained from (1) and (2) respectively.

As it may be seen from Fig. 1 that, in case of $\left(c_{1} / c_{2} \cdot l_{2} / l_{1}\right) \geqq 1$ and $0 \leqq M_{1} / M_{2} \leqq 1$, the velocity $d U_{1} / d t$ becomes zero or negative or equal to the velocity $d U_{2} / d t$ in conformity with the values of $\left(M_{1} / M_{2}\right)$ and $\left(c_{1} / c_{2} \cdot l_{2} / l_{1}\right)$. Concerning these matters, of course, the analytical treatments may be possible, but they will be done in other occasion.

In the next place, the repulsion coefficient $e$ between the two objects may be obtained from (1) and (3) as follows:

$$
e \fallingdotseq \frac{\left[\frac{5}{6}+\left\{1-\frac{1}{6}\left(\frac{c_{1}}{c_{2}} \frac{l_{2}}{l_{1}}\right)^{2}+\frac{1}{2 \pi^{3}}\left(\frac{c_{1}}{c_{2}} \frac{l_{2}}{l_{1}}\right)^{3} \sin \left(2 \pi \frac{c_{2}}{c_{1}} \frac{l_{1}}{l_{2}}\right)\right\} \frac{M_{1}}{M_{2}}\right]}{\left[\frac{7}{6}+\left\{1+\frac{1}{6}\left(\frac{c_{1}}{c_{2}} \frac{l_{2}}{l_{1}}\right)^{2}-\frac{1}{2 \pi^{3}}\left(\frac{c_{1}}{c_{2}} \frac{l_{2}}{l_{1}}\right)^{3} \sin \left(2 \pi \frac{c_{2}}{c_{1}} \frac{l_{1}}{l_{2}}\right)\right\} \frac{M_{1}}{M_{2}}\right]} .
$$




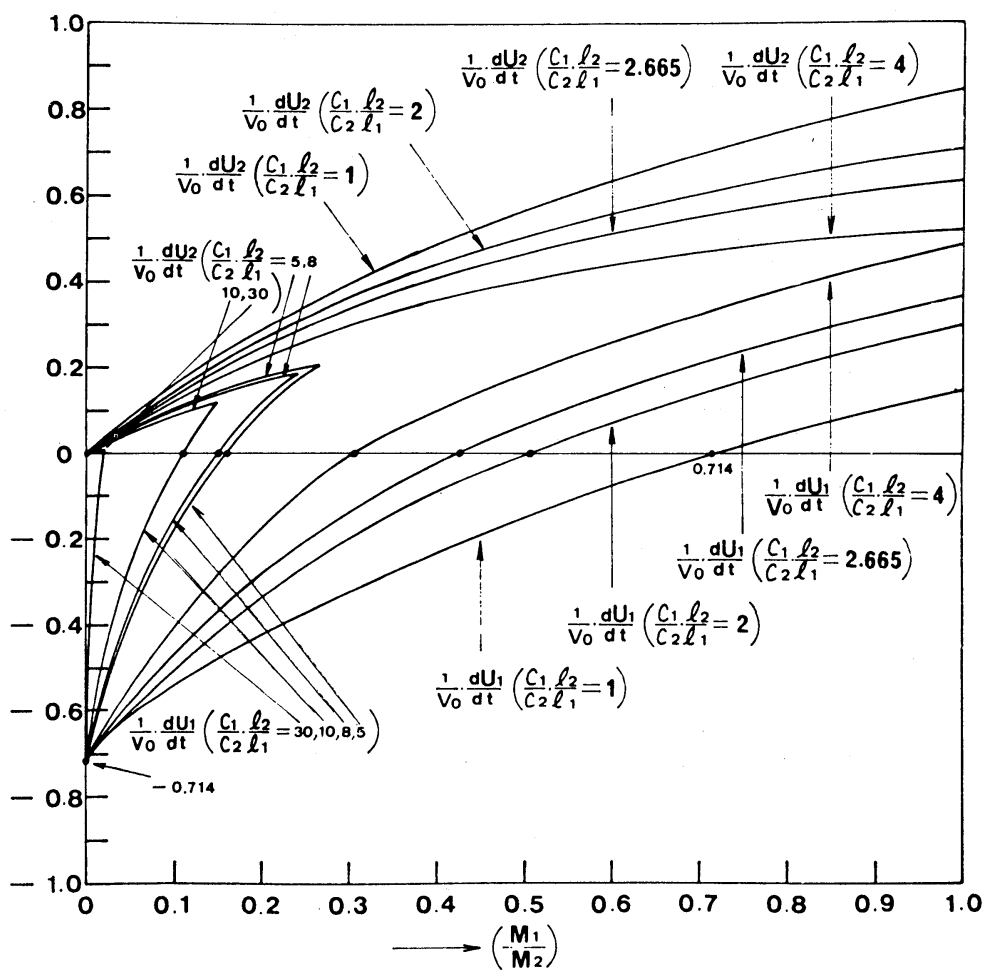

Fig. 1

Now, the collision in case of $e=1$ is commonly called a perfectly elastic collision, but the expression (5) shows that, in case of $e=1$, collision cannot be existent, because of the fact that the following relation must hold good in case of $e=1$.

$$
\frac{M_{1}}{M_{2}} \fallingdotseq-\frac{1}{\left(\frac{c_{1}}{c_{2}} \frac{l_{2}}{l_{1}}\right)^{2}\left\{1-\frac{3}{\pi^{3}}\left(\frac{c_{1}}{c_{2}} \frac{l_{2}}{l_{1}}\right) \sin \left(2 \pi \frac{c_{2}}{c_{1}} \frac{l_{1}}{l_{2}}\right)\right\}} \text {. }
$$

For example, for the case when $\left(c_{1} / c_{2} \cdot l_{2} / l_{1}\right)=1$, the expression (6) shows that the value of $\left(M_{1} / M_{2}\right)$ becomes equal to -1 . Therefore, it can be seen that a perfectly elastic collision corresponding to the case $e=1$ is unable to be existent in the present collision problem.

In the next place, the collision in case of $e=0$ is called ordinarily a non-elastic collision. For example, in case of $\left(M_{1} / M_{2}\right) \doteqdot 0.275$, and $\left(c_{1} / c_{2} \cdot l_{2} / l_{1}\right)=5$, as shown in Fig. 1 , both the velocities $d U_{1} / d t$ and $d U_{2} / d t$ become equal to each other, and moreover this fact corresponds to $e=0$. And, therefore, this fact needs not necessarily be called a non-elastic collision.

As concerns coming to a stop of the object $M_{1}$ subsequent to the collision, the following relation may hold in a good approximation. 


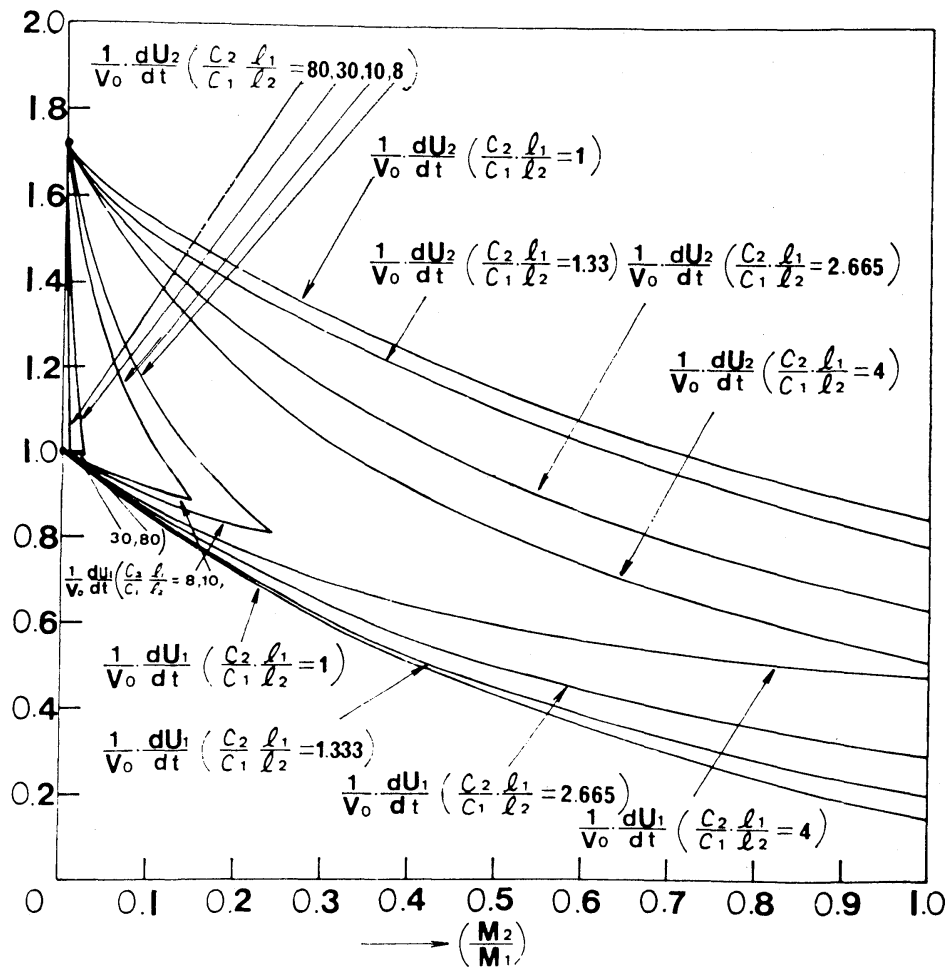

Fig. 2

$$
\frac{M_{1}}{M_{2}} \fallingdotseq \frac{1}{\left[6+\left(\frac{c_{1}}{c_{2}} \frac{l_{2}}{l_{1}}\right)^{2}-\frac{3}{\pi^{3}}\left(\frac{c_{1}}{c_{2}} \frac{l_{2}}{l_{1}}\right)^{3} \sin \left(2 \pi \frac{c_{2}}{c_{1}} \frac{l_{1}}{l_{2}}\right)\right]} .
$$

Then, taking $\left(c_{2} / c_{1} \cdot l_{1} / l_{2}\right)$.in abscissa, $M_{1} / M_{2}$ in ordinate, it is able to obtain the curve of the condition of stop $\left(d U_{1} / d t\right)=0$, and both regions of $\left(d U_{1} / d t\right)>0$ and $\left(d U_{1} / d t\right)<0$. In this case, of course, the relation $0 \leqq\left(c_{1} / c_{2} \cdot l_{2} / l_{1}\right) \leqq 1$ (or $\left.\infty \geqq\left(c_{2} / c_{1} \cdot l_{1} / l_{2}\right) \geqq 1\right)$ holds good in both objects.

Next, the relation $\left(c_{1} / c_{2} \cdot l_{2} / l_{1}\right) \geqq 3.91(\doteqdot 4.0)$ is the condition, in which both the velocities subsequent to the collision become equal to each other when $\left(c_{1} / c_{2} \cdot l_{2} / l_{1}\right) \geqq 1,0 \leqq\left(M_{1} / M_{2}\right) \leqq 1$. The condition abovementioned is, of course, obtained under the permissible assumption that $\sum_{\nu=1}^{\infty} 1 / \nu^{3} \cdot \sin \left(2 \nu \pi\left(c_{2} / c_{1} \cdot l_{1} / l_{2}\right)\right) \doteqdot \sin \left(2 \pi\left(c_{2} / c_{1} \cdot l_{1} / l_{2}\right)\right)$, and moreover, $\sin \left(2 \pi\left(c_{2} / c_{1} \cdot l_{1} / l_{2}\right)\right) \doteqdot 2 \pi\left(c_{2} / c_{1} \cdot l_{1} / l_{2}\right)$.

And, therefore, for the case when $4.0>\left(c_{1} / c_{2} \cdot l_{2} / l_{1}\right) \geqq 1$ and $0 \leqq\left(M_{1} / M_{2}\right) \leqq 1$, there is no appearance of $d U_{1} / d t=d U_{2} / d t$ between the two objects. Further, it should be added that, in case of $M_{1} / M_{2}=0$, both velocities become $d U_{1} / d t=-0.714 V_{0}$ and $d U_{2} / d t=0$ respectively, in spite of the value of $\left(c_{1} / c_{2} \cdot l_{2} / l_{1}\right)$. 